\title{
Percentage of Persons of All Ages Who Delayed or Did Not Receive Medical Care During the Preceding Year Because of Cost, by U.S. Census Region of Residence* - National Health Interview Survey, $2015^{\dagger}$
}

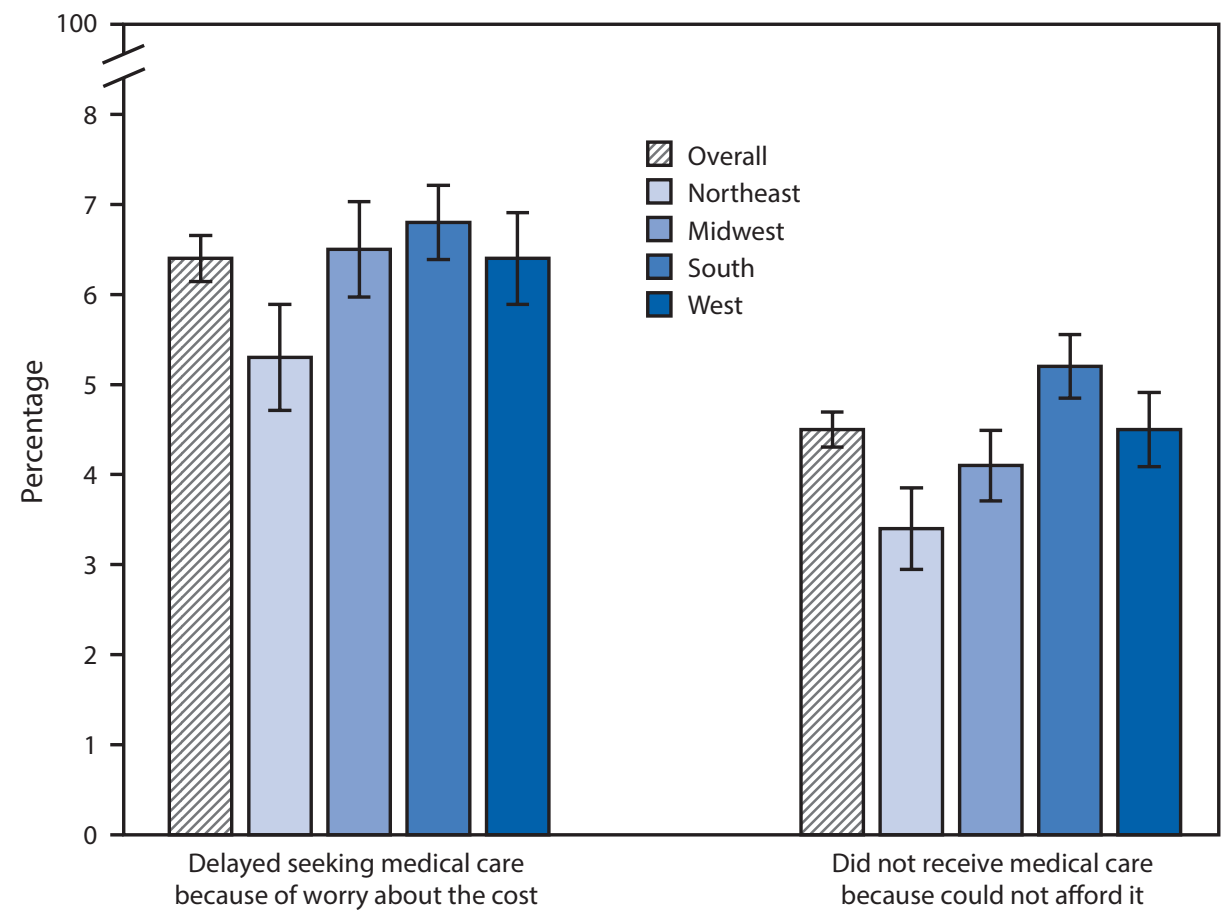

Result of cost concern

\begin{abstract}
* Percentages shown with 95\% confidence intervals. Based on responses to the following questions: "During the past 12 months, has [person] delayed seeking medical care because of worry about the cost?" and "During the past 12 months, was there a time when [person] needed medical care but did not get it because [person] could not afford it?" Both questions excluded dental care. Respondents were asked questions regarding themselves and all other family members of all ages living in the same household. It was possible for a person to have both delayed seeking medical care because of worry about the cost and not received medical care because they could not afford it.

+ Estimates were based on household interviews of a sample of the civilian, noninstitutionalized U.S. population and were derived from the National Health Interview Survey Family Core component. Unknowns were excluded from the denominators when calculating percentages.
\end{abstract}

In 2015, approximately $6 \%$ of persons of all ages (20.1 million) in the United States delayed medical care during the preceding year because of worry about the cost, and 4.5\% (14.2 million) did not receive needed medical care because they could not afford it. Persons living in the Northeast were significantly less likely than persons living in the Midwest, South, or West to delay or not receive needed medical care. Persons living in the South were significantly more likely to not receive needed medical care than those in the Northeast, Midwest, or West.

Source: National Health Interview Survey, 2015. https://www.cdc.gov/nchs/nhis.htm.

Reported by: Jacqueline B. Lucas, MPH, Jacqueline.Lucas@cdc.hhs.gov, 301-458-4355. 